\title{
DETC2010-वास्पर
}

\section{MULTI DOMAIN DESIGN: INTEGRATION AND REUSE}

\author{
Krijn Woestenenk ${ }^{*}$ \\ k.woestenenk@ctw.utwente.nl \\ Hans Tragter \\ G. Maarten Bonnema \\ Design Engineering \\ Faculty of Engineering Technology \\ University of Twente \\ Enschede, 7500AE \\ The Netherlands
}

\begin{abstract}
Design of mechatronic systems is becoming increasingly complex. Companies must continuously reduce time-to-market while increasing the quality, diversity, and functionality of their products. As a result, more and more specialists from various domains are needed to develop such products. To reduce timeto-market, many companies look to reducing the time it takes to design a product. Many focus on the reuse of design objects, leading to libraries of templates and standard components to speed up their design process. However, these reusable design objects are developed and maintained in the specialists' domains, resulting in communication and integration issues between these domains. This paper discusses these issues and proposes a combined approach for model reuse, design integration, and communication between the designers, design tools, and models involved. A case study at a multi-national company successfully demonstrated that the approach leads to a faster and more consistent design process.
\end{abstract}

\section{INTRODUCTION}

Many modern products combine components from mechanical, electrical, and software domains. These are called mechatronic products. This paper tries to reconcile two major challenges in mechatronic system design: complexity of integration and design reuse.

Integration. The development of such products requires the joint effort of engineering disciplines from various domains [10]. This integration poses a problem, because each domain

\author{
Andrés A. Alvarez Cabrera \\ a.a.alvarezcabrera@tudelft.nl \\ Tetsuo Tomiyama \\ Intelligent Mechanical Systems \\ Faculty of $3 \mathrm{mE}$ \\ Delft University of Technology \\ Delft, 2628CD \\ The Netherlands
}

has its own specialists, design tools, and modeling languages. Every new generation of products has a greater product variety and added product functionality, increasing the complexity of integrating the design work into a working system.

A concurrent approach in mechatronic system design requires a shared multidisciplinary architecting method to integrate the work from the disciplines, as is demonstrated in a survey in 140 enterprises by the Aberdeen Group [1]. System architecting works more effectively when it is supported by cross-domain tool integration, abstracted multi-domain system representations, and by verification and validation methods. The basis of our architecting method is a shared and common view of the system, understood by all disciplines. This common understanding is captured in a high-level architecture model which is expected to provide a clear system overview for the designers [13]. The architecture model is also used to specify which critical system information has to be transferred between the various existing tools.

Reuse. Time-to-market constraints drive the need for reuse and standardization of design artifacts and knowledge into reusable design objects. According to Gautam [2], reuse can attack a number of wasteful design activities compared to designing from scratch: There is no re-inventing the solution, therefore, associated errors and consistency problems are evaded. Redundant design tasks are prevented, for example, validation and verification for a certain design object can be standardized and reused too. Reusable objects prevent multiple versions of equal solutions, limiting problems when integrating the object into a larger system. The hand-off of information is pre-determined by the interface definition of the objects, which

\footnotetext{
${ }^{*}$ Corresponding author
} 
further reduces transaction waste and waiting time compared to a situation where a designer has to uniquely identify and explain how the design object should be integrated. Finally, because of the fact that the reusable objects are so well-defined, they can often be supported by automation, stored in libraries, and implemented automatically, greatly reducing design time.

Reusable design objects do not only cover structural artifacts of the system such as models for bills of material, geometry or software classes, they can also be developed for models for behavior analysis, simulation, verification, and validation as is shown in, for example, the multi-representation architecture of Peak[3]. Basically, reuse concerns generating models. As such, reuse is one of the cornerstones of modelbased engineering.

From the text above we can observe an overlap in complexity issues and reuse solutions. Defining a reusable design object requires a good understanding of the underlying artifact, making it less complex. However, system architecting should provide the appropriate decompositions and interface definitions for the reusable design objects in the first place. Complexity still remains when considering that the design objects evolve over time, as technologies change and design knowledge grows. In single domains, the decompositions and interfaces can be defined more easily than in multi-domain situations. Therefore, this paper will discuss problems around complexity and design integration of reusable design objects in multi-disciplinary situations. For the sake of clarity, we will use the term 'building blocks' to distinguish the multi-domain reusable design objects from the mono-domain ones.

The problem definition section will state a number of issues found in current multi-domain design practice that relate to reuse and integration, as well as provide a review of related literature. This leads to our approach section, in which it is explained how we want to use an architecture model and layered framework in which to formulate and use building blocks.

A case study at a multi-national company provides a proof of concept for approach.

\section{PROBLEM DEFINITION}

The authors work in the "Automatic control code generation for mechatronic systems" research project. This project has industrial partners that represent a cross-section of complex product development, including medical equipment, wafer steppers, printer/copiers, and baggage handling systems, as well as world's leading developer of technical computing software. Interviews and discussions with chief scientists (2), systems and software architects (6), System, software and mechanical engineers $(\sim 10)$, software developers for design and/or integration (6), and a workshop (open discussion 20) [14], have highlighted a number of key issues in complex product development. Often, the same issues came to light. Also, the aforementioned Aberdeen Group survey [1,11] supplies us with additional empirical data. These have led to three perspectives for closer analysis of challenges concerning design complexity and reuse by means of building blocks:

- the organizational perspective of designers who have to work with the blocks,

- the model perspective of how to define integrable building blocks,

- the data and communication perspective of how to store, analyze, communicate and transform the data and information involved.

\section{Organizational perspective}

Multi-Domain. The building blocks must be able to reflect the multi-domain character of mechatronic design and must provide an integrated solution for the synthesis of models.

Many approaches tend to capture this multi-domain character by first providing a structural decomposition (design structures) of a system (assembly, component, part...), and then attributing aspects (material, cost...) to these components and subsequently relating those components based on topology, and flow of material, energy, and signals $[15,16]$. However, in our interviews it became apparent that such a single structural decomposition is undesirable and even unworkable. Try to merge a software view with a Design for Assembly view, and then with 20 other views. Methods to derive an (optimal) decomposition are available [17]. However, is it really necessary to have a single decomposition?

Overview. To integrate their work, the individual designers must be able to 'see' how their contribution fits in the context of the whole design and propagation of changes [11]. Various methods for keeping overview, and integration of these methods, is described by Van Beek in [18].

Use Existing Tools. Clearly, building block development and use must be carried by the engineers who work with them. Specialists want to design within their known domain tools and models, not in some new design tool [1]. Therefore, the domain-specific information must be connected to the building block through the tools and models of the domain specialists. Communication must then be facilitated by frameworks as discussed in the communication perspective.

Simple Definition. Defining new blocks should be made as straightforward as possible. The engineers who work with them must be part of block development [19]. Furthermore, there must be an expectation that the building block will be reused sufficiently to justify the investment in its development, as learned by Rommes and Wijnstra [20].

\section{Model perspective:}

Align to Functions. Reusable design objects are generally made for domain-specific applications, such as a block of 
control code for a motor or a geometric conveyor assembly model. However, this has the disadvantage that the objects often lack a clear relation to high-level architectural requirements or functions. As a result, it can become difficult to (automatically) apply these design objects directly from a high-level input model. Therefore, it is useful to make objects as functionoriented as possible. Suh [21] describes how to link functions to design parameters that can be used in individual models.

Integration. There must be an integrative aspect to group reusable design objects from different domains into one block. This aspect must be common to multiple systems or components in order to serve as a delimitation of the re-usable building block. Integration can be done on the knowledge level as in meta models [22], or in product data management software, or many possibilities in between those extremes.

N:M Mapping. Integration and modularization can conflict. This will mainly be the case when a reusable design object is based on a spatial or structural decomposition. For example, a strictly modular design would have one processor for each software-driven function. This is, of course, economically undesirable. At an architectural level, a component can have multiple functions, and a function can be distributed over multiple components. This must be taken into account by allowing multiple mappings.

Synthesis of Domain Models. The building blocks must provide enough information to allow the construction of domain-specific models. Think of models for analyses and validation, bill of materials, geometry, and software generation. A straightforward approach would be to capture all domain knowledge and data into a single ontology and language. A 'Model of Everything' might solve the integration challenge but create new problems - it would be laborious to create and maintain [12]. Constructing such a model would take a lot of time, and all those engineers and designers involved would need to learn both the new model language such as the one provided by Nayak [7], and how to use the associated tools. Another approach is letting third party tools generate the models. In that case, only the mappings with an architectural model need to be communicated, as is done in the ToolNet Project[27].

Freedom vs. Speed. Clearly, design freedom, and speed \& consistency conflict with each other. Good building blocks decrease the time needed to model a system, and a block will always be implemented from a library in a predictable and consistent way. However, the contents of the block are not easily changed when a situation calls for it - the design freedom is restricted to the various configurations allowed by its parameterization. Furthermore, every technology changes: components get updated, tools are replaced, and new design methods are implemented. Pre-defining the parameterization of a building block means it cannot be re-defined without risk. Each block has been engineered and tested for integration in a certain context. If the block is changed, there is no certainty that it can still be integrated into a new system without errors. The building blocks must be constructed in such a way that monodomain components can be replaced or updated without throwing the entire block away. Version control of the blocks must then be applied to ensure consistency. In [28] Scherer defines a procedure to keep parametrically defined chains of models consistent during changes.

\section{Data and communication perspective:}

Capture Design Decisions. Communication between various domains can be realized through meetings [13]. At these meetings, project overview and progress, the system decomposition and interfacing, work integration, and other design decisions are discussed. Often, the results are documented in text documents and stored in an archive, never to be seen again. A living architecture model can be maintained, and through a communication framework, can be used to store and keep track of these design decisions, making information accessible for everyone online. Reverse engineering existing design decisions is a laborious but often very insightful process for a company, as Borches explains in [23].

Share Information. Building blocks consist of data from multiple sources. Because mono-domain models and software tools cannot always communicate with each other directly, modeling using building blocks must rely on an effective communication framework for the proper integration and transformation of the data and information. Literature provides many such frameworks: DOME[24], IDIMS[25], TENT[26] to name a few. Also, advances in software development practices provide us with the standards to make our own tooling: DSL, UML, SysML, OWL, CORBA, XML, TCP/IP etcetera...

Impact Analysis. When we consider a company employing a hundred engineers and designers all working with, and expanding libraries of, reusable design objects, we can see that managing such libraries can become a problem. If someone rewrites a block of control code for a certain motor control, that person perhaps does not know that that software component is also used in another motor-control design object. The libraries of the multi-domain building blocks should be checked for consistency of their mono-domain reusable design objects. Nayak [7] describes how to formulate and check these impacts.

Lean Models. Automatic modeling of a system's model using building blocks must go fast: designers tend to dislike waiting on computers, even if a lot of calculations need to be made. This means the building blocks should be as 'lean' as possible. The building blocks should contain just enough (but no less) information to describe an implementation. Much domain-specific model information can be made available through referencing, instead of keeping it locally within a 
building block, thus separating the multi-domain modeling part from the mono-domain part.

Platform Development and Support. In the ideal case, a dedicated department for developing technology for the analysis and synthesis tools will be needed to automate, maintain and improve the design process, and support communication across the disciplines. These product independent investments over the long term are often not well "sold" to higher management [4]. Therefore it is necessary to be able to 'retro-fit' a building block method on existing modeling systems without too much addition of new tools. The company involved with our case study, however, has spent the previous ten years developing their technology platform for reusability. In their case, this development was certainly worth the effort, as the case study will explain. Muller [29] describes some trade-offs involved in deciding when to start technological standardization.

As could be seen from the previous section, a lot of methods, frameworks and tools are already present. Our approach therefore does not center its focus on yet another integration framework, but more on how to develop the building blocks themselves in relation to their architectural goals.

The framework needed to use, implement and maintain the blocks will be describes as short as possible. The three perspectives discussed above have pointed to a number of key challenges that need to be addressed:

- communication between designers, tools, and models must be supported,

- reusable design objects need an integrative aspect to be usable in a building block,

- the (re-)development time for building blocks must be minimized,

- consistency of models, building blocks and libraries must be maintained.

\section{APPROACH}

This approach describes tools to handle complexity challenges and speed up the design process through reuse. We focus on choosing the right abstractions of the functionality, requirements, design aspects, structure, and behavior of a system. By choosing the right abstractions, we can define a mechanism that is able to generate models in multiple domains from a central architecture model.

This section begins with specifying a basic architecture with which the design with building blocks takes place. Then the details of the key challenges are analyzed. We conclude that a functional delimitation of building blocks can help structure a design process and improve communication between the designers, tools and models.

The automated synthesis of models will be discussed, using the proposed abstractions. Finally, the combination of the topics above leads to a framework in which various design processes can be executed and supported automatically.

\section{Architecture}

The design process using building blocks takes place with a system architecture consisting of an architecture layer, a monodisciplinary layer, and a communication layer between those.

This means a system is described at various levels of abstraction to condense the amount of information and improve overview. See in the example in Figure 1 how the amount of information grows while the design choices are already fixed at the architecture layer. This example will be referred to throughout the approach section: The communication layer translates information from an architecture model to the domain tools that instantiate the domain models. The architecture model contains the representation of the building blocks to make mapping possible. The chosen synthesis method shown is a Visual Basic API script for the domain tool, but other means of building block implementation are also possible. Other domain specific reusable design objects can be mapped to the building blocks in a similar fashion.

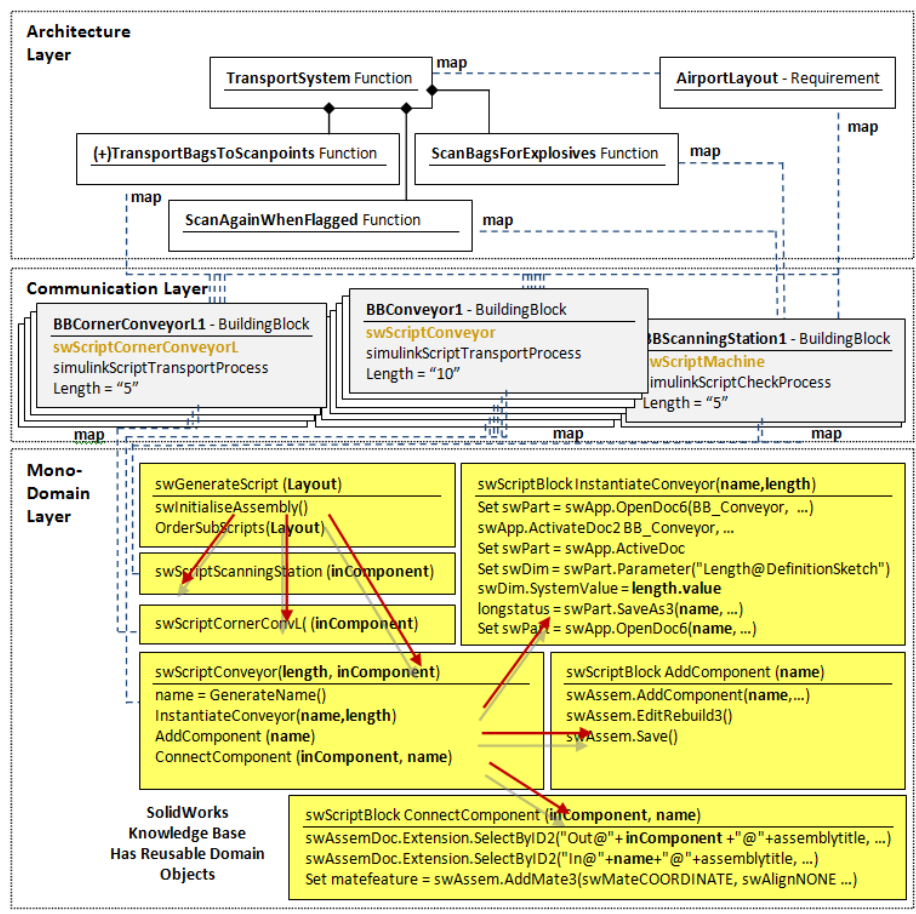

Figure 1. AN EXAMPLE OF HOW THE REUSABLE DESIGN OBJECTS OF A SYSTEM ARE CONNECTED TO THE ARCHITECTURAL MODEL.

The system's modeling will take place in the architecture layer. Building blocks are selected to perform certain functions (or purposes, or goals). Requirements are defined for these blocks, specifying the values needed for implementation. The building block will capture the mappings to the connected mono-disciplinary reusable design objects and to the methods to implement these. 
In the mono-disciplinary layer, we have libraries of monodisciplinary reusable design objects, which, for each domain, can be composed into the mono-disciplinary models by automated model transformation. Apart from structural models such as geometry models (as is depicted in Figure 1), we can also compose analysis and simulation models, software instructions, electronics layouts, cost calculation models, etcetera.

\section{Communication between designers, tools, and models}

As mentioned earlier, a building block must be a reusable part of the design, and for this, the use of a building block must be understandable to the designer. The ideal delimitation of the building block can differ between specialists of the various domains: A mechanical engineer could see a decomposition of geometric shapes as an ideal delimitation, an electronics specialist sees a decomposition based on logical schemas and a software specialist sees a decomposition based on class diagrams. These views are not reconcilable by choosing one of the specialists' sides. If the domain decompositions were the same in every domain, the system would be perfectly modular, which is mostly not the case, or even unwanted when considering the extra cost and effort to design it as such.

The answer that the authors found in the present work is that functions provide the building blocks with a good delimitation. Thus, a building block for a routine design situation must have a well-defined function in a system. An example from our case study illustrates this clearly: A baggage handling system is formed by combining parts that accomplish functions such as "transport bags to checkpoint", "separate bags in two vertical levels", and "scan a bag for explosives". Appropriate building blocks that accomplish these functions are a "conveyor", a "vertical sorter", and a "scanning station". Often, these building blocks will correspond to systems composed of solutions pertaining to several design domains, but this depends on the type of system being designed. See Figure 1 for a geometry domain example.

This does neither mean that each building block must have only a single function, nor that a function cannot be defined across several building blocks. There is no invariable one-toone mapping between functions and building blocks. Continuing the previous example, building blocks such as the "conveyor" can, at the same time, act as a buffer to accomplish the function "store bags on hold". A higher level function, such as "transport bags to scanpoints", may need to be carried out by several lower-level functions such as "transport straight" and "transport corner", which in turn are accomplished by certain "straight line conveyor" or "curved conveyor" building blocks.

Functions should be concepts common to all the personnel involved in the design of a particular kind of system. In that way, functions form a language:
- that can be effectively used to bridge communication barriers among designers during design,

- that can serve as an indexing mechanism to inquire and search building blocks to compose a design.

The functional decomposition of a system, mapped to a set of building block instances, combined with a set of requirements can serve as the basis for an architecture model. This architecture model is the communication backbone of the design process. On one hand, we can use it as an input model for (automatic) synthesis of various domain-specific models through reusable design objects. On the other hand we can use the architecture model to validate the generated output models, by checking whether the system design fulfills the intended goals as specified by the requirements and functions.

\section{Integration between domains}

When a function is defined that is understood by all involved, it can also be used as an integrative aspect between the various design domains. In a single design domain, it is often not so difficult to define reusable design objects. (For example: parameterized geometric components in a drafting CAD tool, matlab .m script for the generation of a behavior model). It must be noted that single-domain models rarely offer a self-contained implementation of a solution, as they just represent a limited part of a solution that involves several disciplines.

With design objects in multiple domains, it is often not apparent what their commonality is. That can make it difficult to formalize building blocks. We propose using the functionality of the block as the basis for integration of design objects from multiple domains. Figure 2 shows that different mappings can be made from domain decompositions to the functional decomposition - In our approach, the functional decomposition is the backbone in the design process. Building blocks then become a means to perform certain functions in a system.

To keep with the example of the function "transport straight", and its building block "conveyor" of Figure 1. The conveyor can be viewed as a static structure, but when we add the function, it needs a behavior to fulfill its purpose.

\begin{tabular}{|c|c|c|}
\hline $\begin{array}{c}\text { Simulink } \\
\text { Decomposition }\end{array}$ & $\begin{array}{c}\text { Function } \\
\text { Decomposition }\end{array}$ & $\begin{array}{c}\text { SolidWorks } \\
\text { Decomposition }\end{array}$ \\
\hline +TransportProcess & + TransportSystem & +ConveyorAssembly \\
\hline --+NormalRunning & --CheckinPoints & -CheckIn1 \\
\hline ----ContinuousTransport & --+TransportBagstoScanpoint & -+Conveyor 1 \\
\hline ----RegisterBag ..... & ----Transport Straight (x6) & -..-PLC_Unit \\
\hline --.-TimedCheckPerBag (x & --.--Transport corner (x4) & -.---Motor \\
\hline -..-DispatchBag ...... & ---ScanBagsforExplosives & ..... \\
\hline$\cdots+$ StartUp & --ScanAgainWhenFlagged & $\cdots c$ \\
\hline -.--InitialiseConveyors & ---StoreBagsOnHold & $\ldots$ \\
\hline -..-CalibrateScanners & -.-PlaneLoadingPoints & -.. \\
\hline --+EmergencyShutdown & +SafetyShutdown & --ScanMachine \\
\hline -..-StopScanners & $\ldots$ & -..-Motherboard \\
\hline
\end{tabular}

In practice, see Figure 2, this could mean that the software domain must supply control code "ContinuousTransport" for a Programmable Logic Controller (PLC), which gives an 
electric motor from the mechanics domain a rotating instruction, through electronic connectors and busses developed by the electronics domain. As such, the function is the integrating aspect between mechanic, electronic and software components.

The mono-domain components, such as the PLC code or electric motors, can in turn be defined as instances of reusable design objects, which means we can define layers of reuse. If we look again at Figure 1, we can see that the mono-domain component "swScript AddComponent" is not limited to the conveyor geometry. This enables the composition of building blocks. On the bottom we have the mono-domain libraries. Specialists in that field can organize the reusable design objects according to functional goals. Dedicated transformation tools can generate domain specific models from the higher level input. A level higher, at the communication layer of Figure 1, we find the multi-domain building blocks, defined to accomplish a specified function within certain requirements. These building blocks contain a mapping to all mono-domain reusable design objects needed to fulfill the function, thus integrating the domains: the multi-domain building blocks bridge the logical gap between the abstract architecture model and the various high-detail engineering domains.

\section{(Re-)Development time for building blocks}

As technologies and tools change over time, new or updated building blocks will be needed. The proposed building block definition, with its functional delimitations, layered in multidomain and mono-domain, help simplify their development.

Development of mono-domain reusable design objects. Although products evolve and functionality will be added, it is unlikely that the full set of possible functions or the types of requirements will change very much when upgrading a system, even if technology changes: the function delimitation is more or less stable over time. So when the building block specifies what is needed in order to achieve a functional goal, given certain requirements, this means that the topologies of the building blocks are stable too. For example, in Figure 1, we can change the algorithm within the reusable design object "swScript AddComponent" without changing the overall synthesis process - the resulting models will maintain their functionality.

The function delimitation decouples the building blocks from their implementations. As a consequence, it is possible to keep developing the domain-specific reusable design objects connected to the building block while keeping the block delimitation unchanged - for example, by adding detail to the "BB_Conveyor" reusable object referenced in Figure 1. The decoupling makes developments safer, and faster, because only a small functional part needs to be considered at a time. However, the updated domain objects must be validated to see if the implemented block as a whole can still perform its function within the system once instantiated. The combination of the function delimitation and the requirements-based configuration provide the right context to check this validity.

Development of multi-domain building blocks. In the multi-domain case, changes and additions to building blocks become easier, because we can utilize our own formalisms, based on object-oriented modeling, instead of those of the mono-domain tools, and exploiting advantages like inheritance and encapsulation.

Basically, the multi-domain blocks are mappings from functions and requirements to mono-domain reusable design objects This means we can use collections of references to connect objects. The technology to do this can be largely packaged in Domain Specific Language tools (DSL) like the Eclipse Graphical Modeling Framework (GMF) [5], to isolate the user from difficult new design and programming skills.

As the building blocks convey the list of ingredients needed to perform a function bottom-up, and a set of requirements on how to configure models top-down, it is important to keep these information flows consistent. The composition and validity of the synthesized domain models are out of the scope of the building block; they need to be checked on the mono-domain layer. It helps us to see the network of multi-domain building blocks as a mapping to maximize the reuse of the mono-domain design objects. In other words, to reduce the number of monodomain blocks as to increase standardization. As in Figure 1, use "simulinkScriptTransportProcess" to perform in both "BBCornerConveyor" and "BBConveyor", since the shape of the conveyor is irrelevant to the desired behavior of the connected electric motor.

\section{Consistency of models, building blocks and libraries}

While validation of a design is not easily automated, the communication layer (cf. Figure 3 ) can be used to verify the relations between the functions, requirements, and the building blocks and mono-domain reusable design objects.

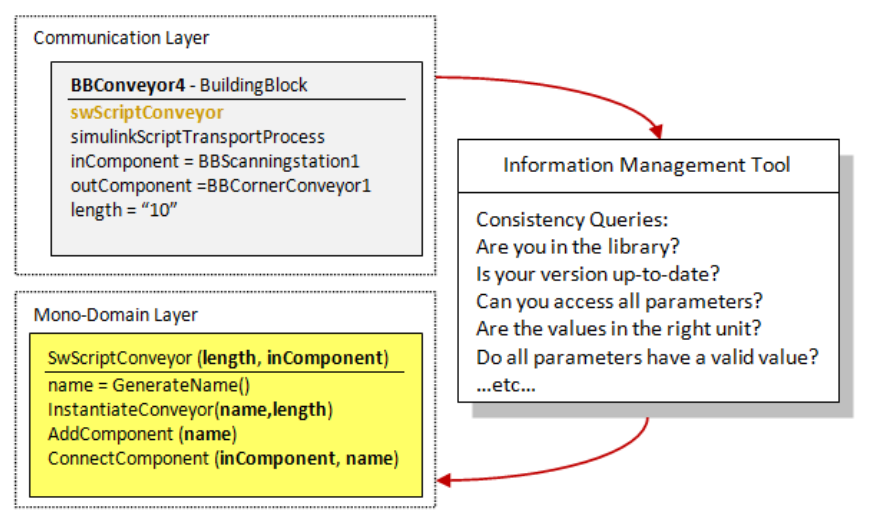

Figure 3. THE COMMUNICATION LAYER ALLOWS FOR THE ADDITION OF INFORMATION MANAGEMENT FUNCTIONALITY.

The communication layer operates on our own formalisms. This allows for the addition of information management 
functionality that is independent of the system or building blocks under consideration, or the types of domain specific tools or models involved. Various relation types, such as parentchild, reference and dependency can be checked on the library level as well as on the model instance level. Basically, the communication layer contains dependency networks that reference data and are navigable with graph and set mathematics [9].

Library consistency. The building blocks provide mappings to reusable design objects in domain specific libraries, as well as parent/child mappings in multi-domain building block libraries. The dependencies of these mappings can be followed analogous to causal relations in a graph, thus enabling impact analyses for design changes. The "AddComponent" block in Figure 1 is used by all reusable design objects of that domain. If someone wants to specialize it to fit better to a specific "swScriptConveyor" object, then the "AddComponent" block runs a risk of not functioning anymore for all other available types - an impact analysis could point out this risk beforehand. For all possible input, the same graph-based search over the library can detect mismatches between the mono-domain libraries - for example to find situations covered in one, but not in all, libraries. A versioning mechanism can check if the referenced information is up-to-date and compatible with the building block. Type-checking of the parameters that are communicated between building blocks, such as the "length" parameter, provides additional consistency-check functionality.

Model consistency. When the building blocks are consistent, the resulting models should be consistent too. That is a matter of model generation in the mono-domains and out of the scope of this approach. However, to construct the models correctly, the multi-domain building blocks must make sure the appropriate mono-domain reusable design objects are referenced. This can become a problem when the libraries are changed a lot over time. Because an architecture model is constructed from functions, requirements and building blocks, the building blocks will be unable to compile the mono-domain models if there is a mismatch between this model and the monodomain libraries. The user will at that point find out there is an inconsistency, and where to look to solve it.

Note that the approach takes into account the verification. Is the information connected? Is it up-to-date? It does not say anything about the validity of the connected information. However, analysis models can be generated with the same method in order to check the validity of the system as a whole.

\section{Synthesis of models}

The whole purpose of the reuse of design information is to generate models from it. These models are synthesized by either composing model fragments and gluing them together, or by rule-based or knowledge-based model generation. The appropriate method will vary from domain to domain and from tool to tool. This paper will not discuss the actual methods of synthesis. However, in Figure 1 we see an example of a script generation for a CAD drafting tool. What we will do is specify the information needed to make any of the methods work.

When functions are used to delimit the reusable - and thus system independent - building blocks, we need system specific information to implement an instance of the block in a system.

When we can use requirements as such specifications, we can make sure the implemented system also follows the requirements - thus assuring compliance to the purpose of the system. In [6], Schotborgh explains how design knowledge can be captured and transformed into automated 'smart synthesis' design tools, as depicted in Figure 4. Note that in our case study as well as in Figure 1 we could isolate topological constraints as the set of requirements, as they were enough to fully define a system.

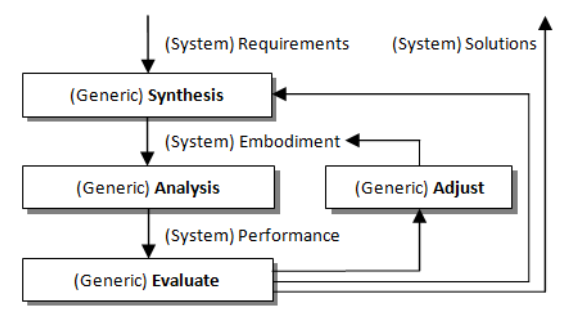

Figure 4. AUTOMATIC SYNTHESIS WORKS WITH GENERIC METHODS FOR SYNTHESIS, ANALYSIS, AND EVALUATION. WITH

THESE METHODS, MANY DIFFERENT SOLUTIONS CAN BE 'EMBODIED', THAT ALL SATISFY THE REQUIREMENTS.

As can be seen in Figure 5, the multi-domain blocks map the functions to the mono-domain objects. The blocks are configured to instances by a set of requirements. From these instances, the output models are generated. Verification makes sure the synthesis process goes as planned, the validation checks if the system behavior conforms to the requirements.

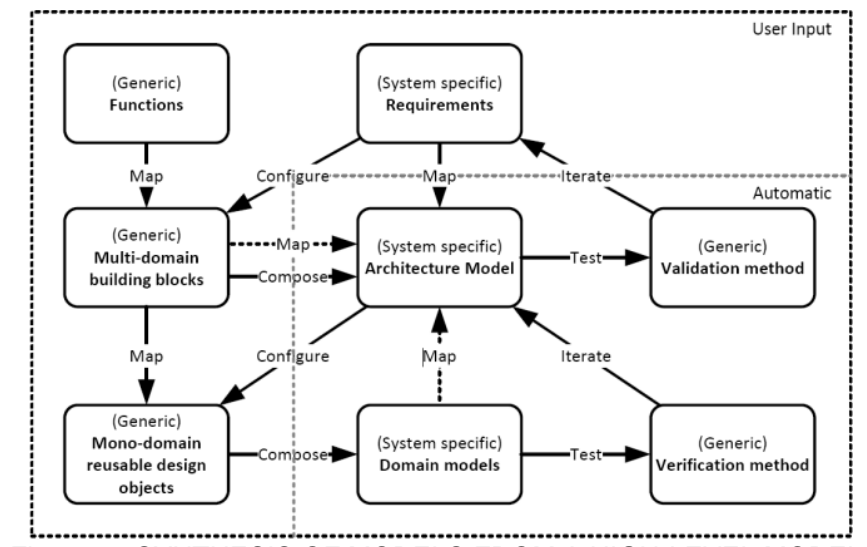

Figure 5. SYNTHESIS OF MODELS FROM A HIGH-LEVEL MODEL THESE MODELS CAN BE FOR ANALYSIS AND SIMULATION, BUT CAN ALSO BE BOM'S, SOFTWARE CODE OR CNC INSTRUCTIONS. 


\section{Communication Framework}

An experimental framework was developed to test the approach. One of these experiments was the case study, described below. The communication framework is a set of software tools that support the design process in the layered architecture as defined in the previous sections. The framework packages information from all layers into machine-readable objects with a generic class definition. This class definition, analogous to the composable objects introduced by Paredis [8], allows for the construction of the information networks needed for the topics discussed in the previous sections. Lutters [9] shows benefits of abstract information networks and operations that can be conducted on them. The generic class definitions and the information networks allows for the development of pluggable tools that can be used to support the information flows in a design process, such as depicted in Figure 6:

- An architecture modeler. To model the functionality, requirements, and selected building blocks. It is used as an overview model as well as an input for automatic synthesis of the domain specific models. This model is used to communicate the design goals to all people involved.

- While the function delimitation of building blocks help the integration of the domains on an ontological level, the communication framework can integrate the flow of information between the framework layers. As can be seen in Figure 1 and 5, the definitions in the architecture model can be translated via model transformation to the domain models while those models can provide key parameter values that can be used to verify or validate the generated system as a whole. Synchronizing parameters across the domains makes sure people work with the same numbers.

- As the design progresses, more and more details of the system are known. The information network can communicate the state of the design process to system engineers by keeping track of design object versions, values of key parameters, and status updates.

- Tools for validation and verification. The information network can be navigated and searched to perform checks such as described in the "Consistency of models, building blocks and libraries" section.

- As a result of the points above, the information flows can be tracked and visualized, independent of the system or models under development, and as such, provide overview for system architects or managers.

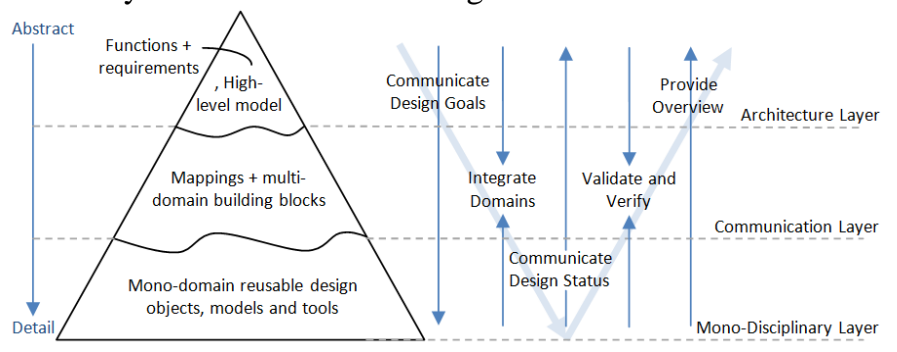

Figure 6. THE FRAMEWORK SHOULD PROVIDE TOOLS TO ENABLE COMMUNICATION THROUGHOUT THE DESIGN PROCESS.

\section{CASE STUDY}

The approach discussed in the previous section was applied in a case study at a manufacturer of baggage handling systems. This company already has a product portfolio based on composing systems from a large set of modular reusable design objects (colored blocks in Figure 7). Mono-disciplinary domain models of hardware, software control, cost calculation, and simulation models are constructed concurrently from these objects. This company expressed a need for a better overview and control over the model generation and the need for a map of the interrelationships between their libraries of reusable design objects. The reason for the needed improvements can be found in the evolution of their design method:

The mono-domain design objects consist of model fragments that can be maintained as data in domain-specific libraries for every engineering domain. A designer or a specialized software tool can then select and configure the correct instances. From an organizational perspective, this has the benefit of leaving the domain specialists responsible for reusable design objects in their own domains. They do not have to acquire the programming skills necessary to build software tools and representations for the building blocks. However, as the libraries grew over the years, the specialists lost oversight over the way the domain-specific libraries interconnect, making manipulation of the libraries risky. Who knows the propagated consequences of one change in just one library?

Therefore, in this case study, a communication layer was introduced between the existing architecture layer and monodisciplinary layer. We data-mined the existing libraries for the reusable design objects as well as all their possible configurations for all possible functionality. The building blocks form a map between the function and requirement of a certain piece of baggage handling system and the various software and hardware needed to accomplish this function. Fortunately, this process could be automated because of the company's previous efforts and attention put into the uniform ontology of the existing libraries. This resulted in a new library of multidomain, function-based, building blocks, defined along the lines explained in the approach section (see Figure 8).

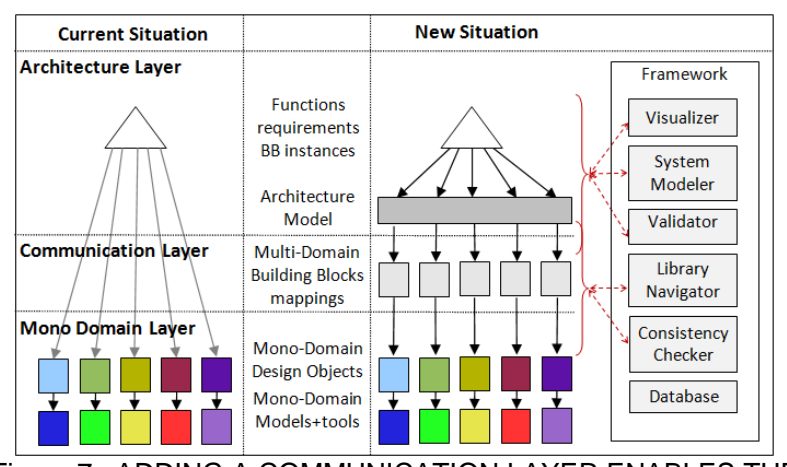

Figure 7. ADDING A COMMUNICATION LAYER ENABLES THE ADDITION OF PLUGGABLE FUNCTIONALITY TO THE INFORMATION FLOWS IN THE DESIGN PROCESS. 
The composable object format, based on [8], allowed us to capture all this information in a graph-based information network in the communication layer. This network can be navigated and manipulated independent of the information inside it, allowing for the addition of modeling and informationmanagement functionality (see Figure 7 and 8).

A company specific modeler was developed during the case study that can be used to build high-level architecture models. These models can then be used for domain-independent analyses. For example, the architecture model can discern between available building blocks and new, and thus unknown, components. The unknown components must still be developed 'manually' (about 20\% for every system). A user can now specify the functionality in the form of a flow diagram and a geometric layout as an input from which all needed software and hardware models can be generated concurrently using the company's existing domain tools. Most importantly, the introduction of a communication layer has led to three main points the company will take over in a new version of the company's design environment:

1. Navigation and visualization of the information network enables impact analysis for design changes: Relations between building blocks and the connected reusable design objects can be followed to see the chain of objects that could be influenced by changing a certain object.

2. Enable discovery of mismatches between the libraries: By looking at the set of reusable design objects connected to a building block, it becomes clear if a piece of information is lacking, is an old version, or is wrongly referenced.

3. Enable discovery of mismatches between a given architecture model and the libraries. The original model generation environment would not give an error if a certain function/requirement combination could not be implemented, the missing reusable design objects would just be skipped. The new environment already specifies the needed design objects in the building blocks, if some are missing or incorrect, then the consistency tool will notice it.

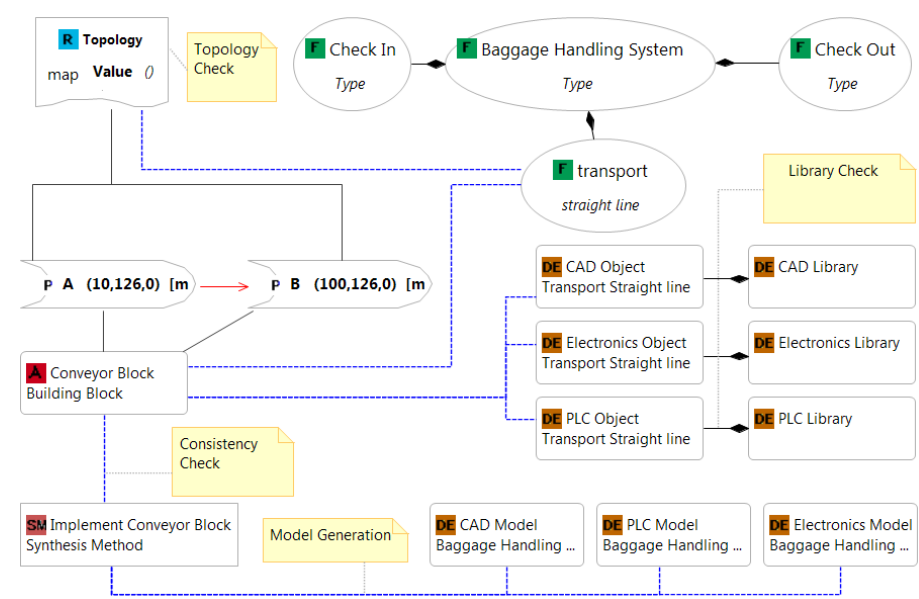

Figure 8. A 'TRANSPORT' FUNCTION IS MAPPED BETWEEN A AND B. A STRAIGHT CONVEYOR IS THE FUNCTION ENABLER.

BLUE = MAPPINGS, BLACK = CONTAINMENT. RED = DEPENDENCY

\section{CONCLUSIONS}

Our case study showed us the approach works in an industrial setting. As stated there, the company in question will take over three recommendations. The architecture model proved to contain enough information to generate all models needed to describe and analyze a system in the case study. It remains to be demonstrated if such function centered models will be equally successful in other situations. This will be researched at other companies, but given the hundreds of elements in the current case, the expectations are hopeful.

The case study does not provide any indication that this approach is better than existing solutions from literature. Unfortunately, benchmarking against comparable frameworks is difficult because software implementations are often not available. However, this approach is not a software tool, but a way of architecting and organizing design information. The added value of the approach is to define a working method to promote integration and reuse in a multi domain design situation.

This we have tried to accomplish by using architectural concerns such as functions and requirements as delimiters for reusable building blocks. As such, building blocks bridge the logical gap between an abstract architecture model and the various engineering domains. Separation of three distinct layers in the design process (architectural, communication, domain) leads to an extendable framework in which ingredients of building blocks can be maintained and composed independent of each other. The same layering allows for system-independent information management functionality in the communication layer, keeping the models and libraries consistent and manageable.

Some lessons learned from the research for this paper will be scrutinized in further research on integration and reuse:

- Aligning architectural goals with reusable objects, all the way down to the domain models, is essential.

- Capture the transformation of architectural goals, through design decisions, into domain model specifications in causal networks of parameters.

- A method for reuse focuses on information needed for generating models, not on describing behavior or structure of systems directly.

- To improve reuse, focus on the design process rather than the design - combine reusable design objects with reusable design processes into 'design process patterns'.

- Separation of information management functionality from system design leads to system independent tools for consistency and communication.

Implementations at other industrial partners are not taken into account in this paper, but they are underway. These already provide indications that our approach is viable in more than one company. As one of the deliverables, a generic architecture modeling tool is underway of which figure 8 gives a preview. 


\section{ACKNOWLEDGMENTS}

The authors gratefully acknowledge the support of Peter Kamps of Vanderlande Industries for his help in the case study as well as the Dutch Innovation Oriented Research Program 'Integrated Product Creation and Realization (IOP-IPCR)' of the Dutch Ministry of Economic Affairs.

\section{REFERENCES}

[1] Aberdeen Group, 2006. The Mechatronics System Design Benchmark Report.

[2] Gautam, N., R. B. Chinnam, et al., 2007. "Design reuse framework: a perspective for lean development". International Journal Product Development, Vol. 4(No. 5).

[3] Peak, R. S., R. M. Burkhart, et al., 2007. "SimulationBased Design Using SysML Part 1: A Parametrics Primer. Part 2: Celebrating Diversity by Example”. INCOSE Intl. Symposium, San Diego.

[4] Hayes, R. H. and W. J. Abernathy, 1980. "Managing Our Way to Economic Decline". Harvard Business Review.

[5] Eclipse Graphical Modeling Framework (GMF), http: / /www.eclipse.org/modeling/gmf/

[6] Schotborgh, W. O., 2009. Knowledge Engineering for Design Automation. University of Twente, The Netherlands.

[7] Nayak, P. P. 1995. "Automated Modeling of Physical Systems". Lecture Notes in Artificial Intelligence, Springer.

[8] Paredis, C., A.Diaz-Calderon, et al., 2001. "Composable Models for Simulation-Based Design”. Engineering With Computers.

[9] Lutters, E., 2001. Manufacturing Integration based on information management. University of Twente, The Netherlands.

[10] Pahl G, Beitz W, Feldhusen J, Grote K.H., 2007. Engineering Design: A Systematic Approach, $3^{\text {rd }}$ ed. London, UK: Springer London Limited.

[11] Aberdeen Group, 2008. System Design: New Product Development for Mechatronics.

[12] Dolk D.R., Kotterman J.E., 1993. "Model integration and theory of models". Decision Support Systems; Vol. 9, pp. 51-63.

[13] Alvarez Cabrera, A. A., Erden, M. S., Foeken, M. J., Tomiyama, T., 2008, "High Level Model Integration for Design of Mechatronic Systems", proceedings of IEEE/ASME International Conference on Mechatronic and Embedded Systems and Applications. Beijing, China. pp. 387-392.

[14] Delft Center of Engineering Design, OPM Lab, 2009. First workshop on complex systems architecting: Best practices and new development. Delft University of Technology, Delft, the Netherlands.

[15]DSMWeb.org. The Design Structure Matrix (DSM). http://www.dsmweb.org/

[16] Tilstra, A. H., C. C. Seepersad and K. L. Wood, 2009. "Analysis of Product Flexibility for Future Evolution Based on Design Guidelines and a High-Definition Design Structure Matrix", ASME IDETC Advances in Design Automation Conference, San Diego, CA.

[17] Tate, D., 1999. A roadmap for decomposition : activities, theories, and tools for system design. Massachusetts Institute of Technology. Dept. of Mechanical Engineering.

[18] T. van Beek and T. Tomiyama, 2008. "Connecting Views in Mechatronic Systems Design, a Function Modeling Approach", Proceedings of IEEE/ASME International Conference on Mechatronic and Embedded Systems and Applications (MESA), pp. 164-169.

[19] Ilan Oshri, Sue Newell, Shan L. Pan, 2007. "Implementing component reuse strategy in complex products environments". Communications of the ACM. Volume 50 (pp. 63 - 67), Issue 12.

[20]Eelco Rommes, Jan Gerben Wijnstra, 2005. "Implementing a Reuse Strategy: Architecture, Process and Organization Aspects of a Medical Imaging Product Family", Proceedings of the 38th Annual Hawaii International Conference on System Science, vol. 9, pp. 312a.

[21] Suh, N. P., 1998. "Axiomatic Design Theory for Systems." Research in Engineering Design.

[22] Kiriyama, T., T. Tomiyama, et al., 1991. "A model integration framework for cooperative design." Proceedings of the MIT-JSME workshop on Computeraided cooperative product development. Cambridge, Massachusetts, United States.

[23] Borches, P. D. and G. M. Bonnema, 2008. "'Living' Architecture Overviews - Supporting the Design of Evolutionary Complex Systems." CIRP Design Conference.

[24] Wronski, J., 2005. A design tool architecture for the rapid evaluation of product design tradeoffs in an Internet-based system modeling environment. Massachusetts Institute of Technology. Dept. of Mechanical Engineering.

[25] Chira, C., O. Chira, et al., 2005. "Multi-agent Support for Distributed Engineering Design." Proceedings of the 18th international conference on Innovations in Applied Artificial Intelligence, pp 155 - 164.

[26] Forkert, T., H.-P. Kersken, et al., 2000. "The Distributed Engineering Framework TENT." Lecture Notes In Computer Science. Vol. 1981.

[27] Freude, R. and A. Königs. 2003. "Tool integration with consistency relations and their visualisation." Proceedings of ESEC/FSE Workshop on Tool-Integration in System Development, Helsinki. pp. 6-10.

[28] Scherer, R. J. and P. Katranuschkov, 2006. "From Data to Model Consistency in Shared Engineering Environments." Intelligent Computing in Engineering and Architecture. Volume 4200.

[29] Muller, G., 2008. "When and What to Standardize; An Architecture Perspective." INCOSE 2008. 